\title{
Gnotobiotics: getting a grip on the microbiome boom
}

\section{Smriti Mallapaty}

The growing interest in the microbiome has driven demand for germ-free and gnotobiotic animals, but facilities are struggling to keep up.

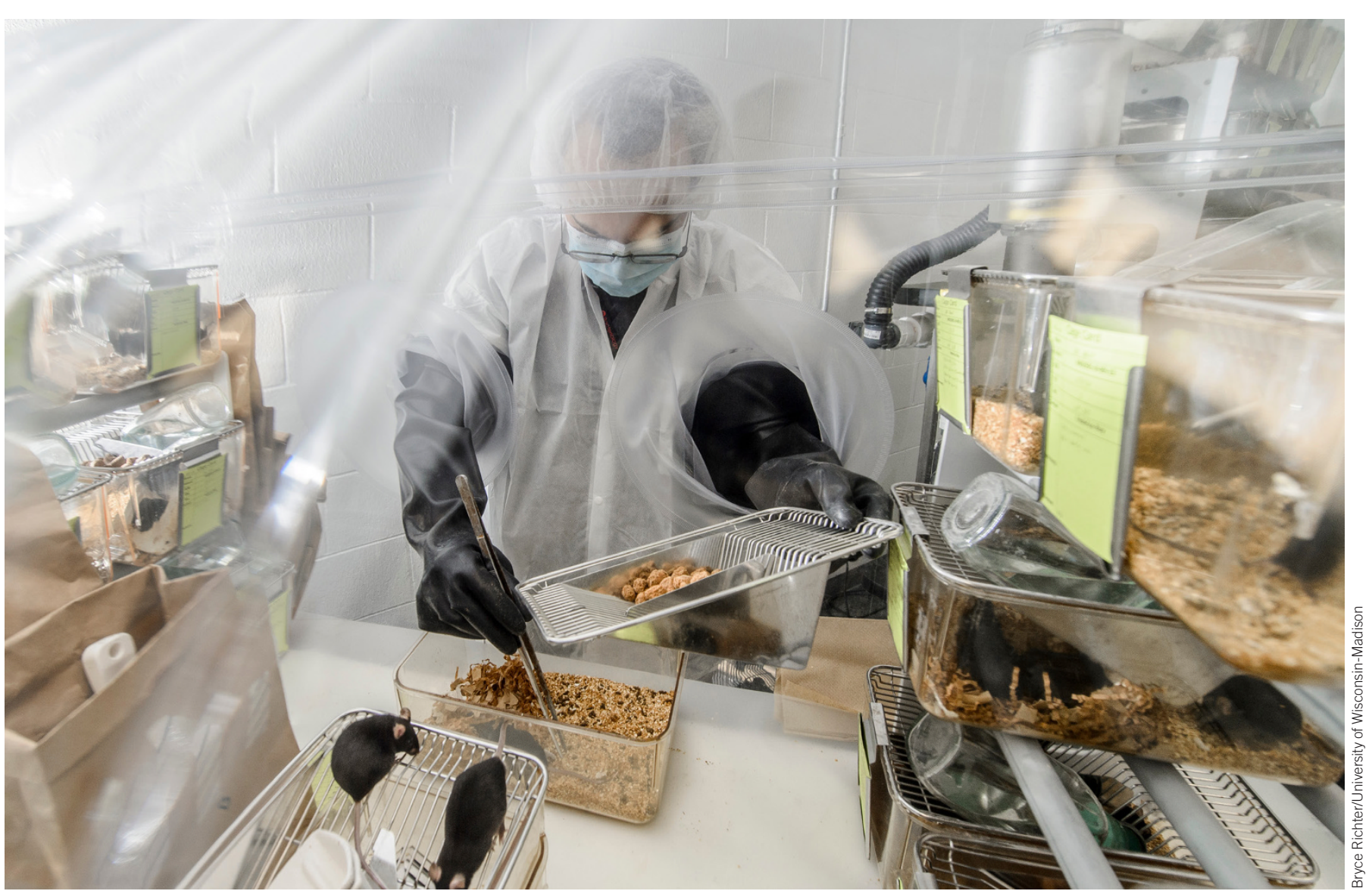

Balfour Sartor was aware of the dangers he posed to his colonies of rats and mice. Their entire existence rested on maintaining an impervious barrier between him and them. These were no ordinary rodents. They were gnotobiotes-a term derived from the Greek word for 'known' and 'life'-born and bred in plastic bubbles, isolated from a world drenched in bacteria, fungi, and viruses.

Smriti Mallapaty is an editor and science writer in Sydney, Australia (Smriti.Mallapaty@gmail.com).
Withdrawn from all known microbial life forms, or containing only known strains, these animals present researchers with a blank slate to study how, exactly, microbes work with and against the body.

Caring for them is a hypochondriac's dream. "The words compulsive and rigid come up at every single step of the process," says Sartor, who manages a large facility as director of the National Gnotobiotic Rodent Resource Center (NGRRC) in North Carolina, which is funded by the United States National Institutes of Health (NIH).
Everything that enters the animals' chamber-food, water, bedding - has to be sterilized in an adjacent portal. To transfer the goods, technicians slide their arms into thick plastic gloves that reach into the transparent bubble, to access the hermetically sealed portal-a precarious task. A slight nip of the glove by an irate mouse could pose "an instant problem," says Sartor. "If they get your finger, they are probably automatically contaminated just with skin bacteria."

"The five-second rule does not apply here," says Federico Rey at the University 


\section{TECHNOLOGY FEATURE}

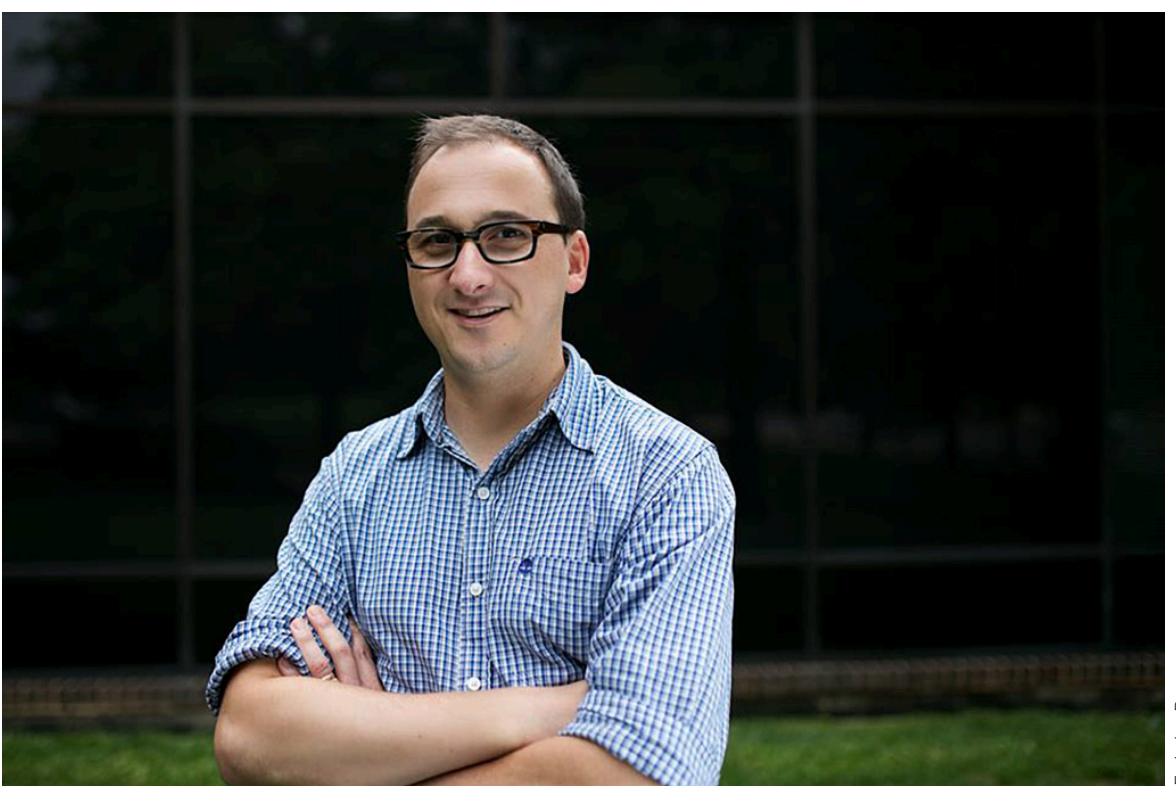

MICROBIAL COMMUNITY ORGANIZER | Federico Rey, Assistant Professor, University of Wisconsin-Madison

of Wisconsin-Madison, who runs his own small facility of 100 mice along with his microbiology lab.

The work can be agonizing and laborious. It demands highly skilled technicians who are fastidious about following protocol and can endure repetitive tasks. Technicians have to "pay a lot of attention to detail," says Rey. "Easy and gnotobiotics don't go together."

While standard vivariums have introduced automated systems for watering, feeding, dispensing bedding, and dumping waste, in a gnotobiotic facility, everything must still be done by hand. Most of the technologies used to raise gnotobiotic animals have not changed since the 1940s, says Sartor. "There haven't been any great new breakthroughs."

Skilled labor is expensive, and so is the time and space needed to fit the bulky isolators. Gnotobiotic animals can cost ten to twenty times more to breed than a standard animal model. As a reference, Sartor's lab employs seven full-time technicians, one embryo transfer specialist, and several supervisors; all to maintain about 100 isolators and distribute 1,600 mice a year. "A large number of lab animal personnel can produce a relatively small number of animals," says Sartor. "If you charged what the animals are really worth, no NIH-funded investigator could ever pay for them out of their grant."
But change is needed, as growing interest in the role of microbes in health and disease has made gnotobiotic models more valuable to researchers.

Between seeping supply and surging demand, the field has reached a critical bottleneck. "The biggest issue is how to possibly make facilities easier to maintain with fewer technicians and less money," says Sartor. "There are some real constraints on size and expense that limit a facility's production."

\section{Gigantic children}

The field of gnotobiotics has evolved over the centuries, together with our understanding of the microbial world. "We humans tend to reinvent things," says Kathryn Eaton, a veterinary pathologist and director of the Germ-Free Mouse Facility at the University of Michigan, set up in 2004 to service the university community.

Soon after Louis Pasteur, the father of modern microbiology, discovered that germs cause disease in the late 19th century, a small group of researchers argued that life could exist, thrive even, without these microbes. Leading the effort was Pasteur's laboratory assistant, Émile Duclaux, who attempted to grow the first germ-free peas and beans ${ }^{1}$. Other European researchers followed with germ-free chickens, guinea pigs and goats. "Question number one was: can animals live without a microbiota?" explains Eaton. The answer was: "Yes, they can." But many of the animals died prematurely.

Eventually, Michel Cohendy, a researcher working under Emile Roux at the Pasteur Institute in Paris, succeeded in raising germ-free guinea pigs in sterilized glass chambers for a period of time long enough to observe noticeable changes in their composure. The germ-free pups grew heavier than the normally raised litter, which Cohendy interpreted as the virtues of aseptic rearing ${ }^{2}$.

His wild conclusions were reported in the Boston Herald in September 1914: "If there were no microbes, men would grow to gigantic stature and have intellectual powers far in advance of those which they possess at present." The New York Times even suggested that "children may acquire strong constitution by similar treatment."

These claims were, of course, false, and researchers later learned that germ-free animals have underdeveloped immune systems. But the animals still offered a useful vessel for studying the role of microbes in the body. Early experiments using gnotobiotic animals, says Eaton, involved simple comparisons between germ-free and normally raised animals, centered on nutrition and immune response - "the vast majority of those studies were biphasic: with 'yes' or 'no' answers,' she says.

In the 1940s, James Arthur Reyniers and Philip C. Trexler at the University of Notre Dame began to design housing chambers that facilitated larger-scale production of germ-free animals. Reyniers invented a bulky but airtight steel isolator, fitted with small windows, ventilation, and rubber gloves ${ }^{2}$. By replacing the steel with plastic, Trexler created a biocontainer model that is used by gnotobiotic facilities to this day.

The engineering feats of Reyniers and Trexler coincided with innovations in the way scientists used animals for research. In the mid-1960s, microbiologist Russell Schaedler at Rockefeller University isolated a group of eight bacterial species-known as the altered Schaedler flora-and began introducing them to germ-free animals to study their effect on the hosts. He chose the bacteria largely because he knew what they 
were-they could be cultivated on a petri dish for closer examination.

By the time Eaton joined the field, experiments involving specific pathogens were commonplace. From just comparing "germfree to not germ-free," researchers began comparing "gnotobiotic with this bacterial community, to gnotobiotic with that bacterial community," she says. The emergence of genomics turned up the complexity on these studies.

\section{Genomics power}

The teeming troves of bacteria living in and on our bodies have long evaded scrutiny due to the limits of traditional culturing methods. But the advent of high-throughput DNA sequencing technology in the 1990s enabled researchers to characterize the genetic makeup of these mysterious forms of matter.

Since then, laboratories all over the world have rushed to take a census of the trillions of bacteria we harbor, and make sense of their roles. Gnotobiotics has been central to this effort. Demand for gnotobiotic technologies dropped from the early 1980s until around 2005, says Betty Theriault, director of the Gnotobiotic Research Animal Facility at the University of Chicago, whose facility supports close to 25 research groups at any given time. "Then, all of a sudden, there was a directive to establish a human microbiome project and there was this scramble to support the research."

In December 2007, the NIH committed \$115 million to the Human Microbiome Project, a five-year project to assess the good and bad behavior of microbial communities in the body. One month later, in January 2008, the European Commission announced its own $€ 22$ million, four-year project, MetaHIT (Metagenomics of the Human Intestinal Tract). In 2016, the US government allocated another $\$ 121$ million for the National Microbiome Initiative, and the Japan Agency for Medical Research and Development agreed to distribute $¥ 1,200$ million to several projects studying the complex relationship we have with our microbial guests.

Over the past 20 years, publications in the field of microbiome science have skyrocketed. A simple search on PubMed for

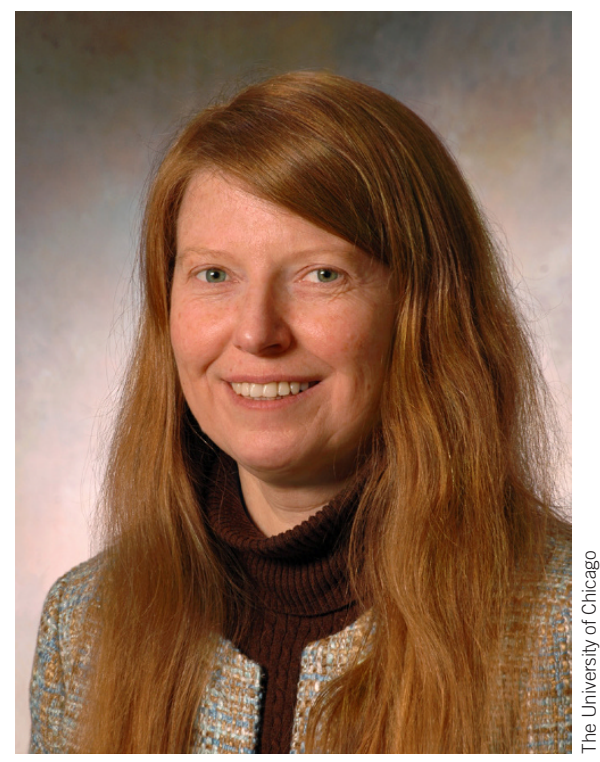

"It is hard to think of any host phenotype that doesn't have a microbial component," says Rey. "Gnotobiotic animals are a powerful tool for testing causality."

\section{A model for growth}

From just a few gnotobiotic labs spread out across the Unites States and the world, including Sweden, Czechoslovakia, and Japan, there has been a "democratization of a large number of places with current technology," says Sartor. Most non-commercial gnotobiotic facilities have run up long waiting lists, and the national facility has even had to turn down some requests due to funding caps, he adds.

The national center offers a four-day training course to help researchers build their own small facilities, which Rey at the University of Wisconsin-Madison benefited from. "Our training activities of groups starting their own facilities have increased rather dramatically," says Sartor. In the past year, the NGRRC has trained technical personnel from 10 universities, the Food and Drug Administration, and the National Institute of Environmental Health Sciences, in addition to providing ongoing technical support to former trainees.

Tapping into the demand for gnotobiotics, in 2015, Eaton and the two technicians who manage her facility in Michigan, Chriss Vowles and Natalie Anderson, published an illustrated how-to on setting up and maintaining a facility, titled Gnotobiotic Mouse Technology. "A lot of laboratories are out there trying to set up their own facilities, and there was nothing to help them out," says Eaton. "The book has certainly been popular."

In 1969, the Japanese non-profit research institute, Central Institute for Experimental Animals, established a gnotobiotic facility, which by 1972 , could produce more than 1,000 gnotobiotic mice and rats a month (their production has since dropped to 100 , as researchers have started breeding the animals in their own labs). "Gnotobiotic techniques are very easily available to Japanese scientists in every field," says Shigeru Kamiya, president of the Japanese Association of Germfree Life and Gnotobiology, and a microbiologist at Kyorin University.

Key individuals in the field have discussed establishing a central database to 
keep track of the available gnotobiotic resources. Currently "each laboratory is a microcosm," says Theriault. Such a support structure could facilitate the sharing of strains between facilities to speed up the time needed to obtain a model, adds Sartor.

\section{Gnotobiotics 2.0}

For all its rigor, gnotobiotics is a field of relatives, rather than absolutes. What counts as a gnotobiotic model today might not be the same tomorrow, as technologies for detecting and characterizing microbes evolve. At the core are sterile, or axenic, animals, devoid of any other living organisms. Here, already, the qualifiers loom. "Right from the start, there is a lot of confusion in the terminology," says Theriault. "A germ-free animal has, to the best of our technology and ability to detect, no organisms associated with it." While technologies for detecting bacteria have advanced rapidly, viruses, fungi, and parasites still remain largely inconspicuous. A puritan might argue, therefore, that the animals are not truly gnotobiotic, given the limits of detection.

Some researchers have even begun to redefine gnotobiotics. Craig Franklin, director of the Mutant Mouse Resource and Research Center (MMRRC) at the University of Missouri, and his colleagues conceived of the concept 'complex gnotobiotics', which by their own admission is not gnotobiotics in the classical sense.

"When most people think of gnotobiotics they think of the germ-free mouse, which has no bacterial microbiota, or they think of a germ-free mouse that has been reconstituted with one or more bacteria, a simplified but controlled microbiota, or more complex human microbiota," says Franklin. "We are coming at it from the other direction. Using ever-evolving next-generation sequencing tools, we can at least partly define very complex microbiota, and ask the broad question-how can we control or manipulate microbiota to optimize the models that we distribute, to address the very real concern with reproducibility of research, where one lab gets one result and another lab can't repeat it."

The idea came from Franklin's experience prior to leading the MMRRC at the University of Missouri. Between 1992 and 2011, Franklin was the director of the

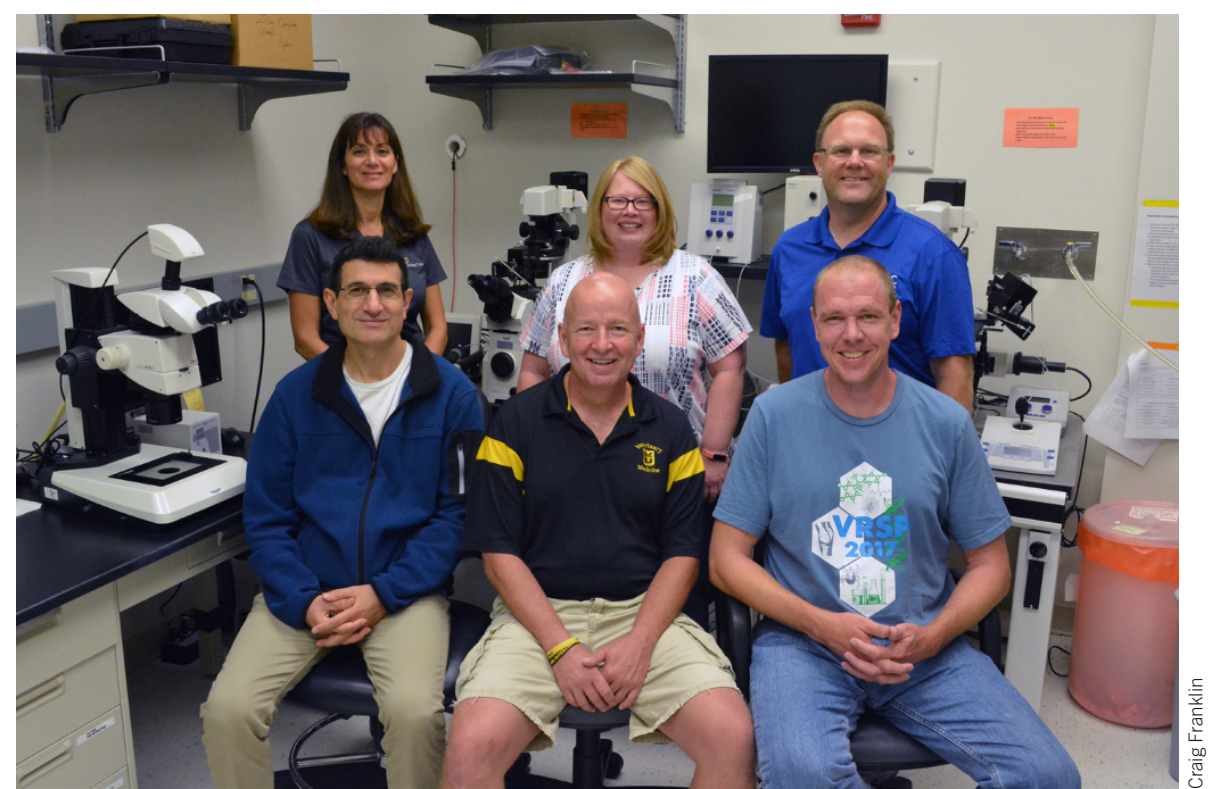

REIMAGINING GNOTOBIOTICS | Craig Franklin (front row, center) and his team at the University of Missouri Mutant Mouse Resource and Research Center (MMRRC)

University's Research Animal Diagnostic Laboratory. Frustrated researchers would send in samples from their animal models to test for the presence of known pathogenic or opportunistic microorganisms. They had recently moved to a new lab, and could not replicate the experiments they had conducted in their previous lab. Often, the diagnostic tests for specific microbes would come out negative, which stirred Franklin's suspicions that differences in the complex mix of microbiota could be a possible contributor.

He confirmed his hunch several years later in a study published in May 2017, which found that mouse models of inflammatory bowel disease raised with different microbiota (representing those present in commercial breeding facilities) had varying levels of disease severity ${ }^{3}$.

At the MMRRC, Franklin has led an effort to create mouse colonies with standardized microbial profiles, which can 'donate' these microbiota to conventional models, giving researchers more control over their experiments, "as opposed to just serendipitously stumbling upon an optimal microbiota." So far, the team has developed four colonies of mice, each with their own special sauce of complex microbiota, which have remained stable for 12 generations.

\section{Small leaps forward}

With automation a distance away, the most noteworthy advance in recent years, says Eaton, has been the development of airtight cages. "The isocages were originally designed to confine dangerous pathogens, until somebody had the idea to flip them from negative to positive pressure airflow, which made them ideal for germ-free models."

The cages can be stacked into space-efficient racks. Each cage serves as an independent unit that can be colonized with different microbes-compared to a typical isolator, which holds about 20 cages, and are all part of the same experiment. "They are much more compact," says Eaton, whose lab has been using them since 2014 . But the food, water and bedding still has to be changed manually.

Facilities are constantly tweaking their protocols to make managing gnotobiotic animals a little bit easier. For example, instead of using Gram staining to detect bacterial contamination, technicians now run PCR tests. Gloves can be changed without taking down the entire isolator. And the filtration systems have also improved. "These are small leaps forward," says Eaton. "I don't know what the next big leap forward will be."

"Researchers are definitely getting more creative," says Rey, especially in developing 
new instruments for analyzing the living animals. Even something as simple as measuring blood pressure, says Rey, currently can't be done easily on gnotobiotic animals without contaminating them. "We all come with our own solutions but as the field becomes more mainstream, new products will emerge," he says. "We are still in the expansion phase."

Will there be breakthroughs? "I'd like to believe so," says Sartor. "But if your next question is what those breakthroughs are going to be, I am strapped to tell you exactly."

1. Reyniers, J.A. Ann. N.Y. Acad. Sci. 78, 3-16 (1959).

2. Kirk, R.G.W. Bull. Hist. Med. 86, 237-275 (2012).

3. Hart, M.L. et al. Front. Microbiol. 8, 792 (2017). 